Article

\title{
A Truncation Scheme for the BBGKY2 Equation
}

\section{Gregor Chliamovitch $^{1,2, *}$, Orestis Malaspinas ${ }^{1}$ and Bastien Chopard ${ }^{1}$}

${ }^{1}$ Department of Computer Science, University of Geneva, Route de Drize 7, 1227 Geneva, Switzerland; E-Mails: Orestis.Malaspinas@unige.ch (O.M.); Bastien.Chopard@unige.ch (B.C.)

${ }^{2}$ Department of Theoretical Physics, University of Geneva, Quai Ernest-Ansermet 24, 1211 Geneva, Switzerland

* Author to whom correspondence should be addressed; E-Mail: Gregor.Chliamovitch@unige.ch. Academic Editors: Sauro Succi and Ignazio Licata

Received: 28 September 2015 / Accepted: 26 October 2015 / Published: 30 October 2015

\begin{abstract}
In recent years, the maximum entropy principle has been applied to a wide range of different fields, often successfully. While these works are usually focussed on cross-disciplinary applications, the point of this letter is instead to reconsider a fundamental point of kinetic theory. Namely, we shall re-examine the Stosszahlansatz leading to the irreversible Boltzmann equation at the light of the MaxEnt principle. We assert that this way of thinking allows to move one step further than the factorization hypothesis and provides a coherent-though implicit-closure scheme for the two-particle distribution function. Such higher-order dependences are believed to open the way to a deeper understanding of fluctuating phenomena.
\end{abstract}

Keywords: kinetic theory; non-equilibrium statistical mechanics; maximum entropy principle

\section{Introduction}

While the formulation of equilibrium statistical mechanics in terms of the maximum entropy ("MaxEnt") principle goes back to Jaynes' seminal work [1,2] in the 50s, the last decade has seen a spectacular revival of this approach. In particular, the MaxEnt-based characterization of complex systems presented in [3] paved the way to applications in a variety of fields ranging from linguistics 
to biology [4-8]. First focussed on equilibrium situations, these works soon turned their attention to non-equilibrium properties as well [9-12].

However, it seems that these authors paid comparatively little attention to more "fundamental" issues. In the present letter, we would like to reconsider some aspects of the kinetic theory of gases at the light of the MaxEnt philosophy. More precisely, kinetic theory relies heavily on the so-called Stosszahlansatz which asserts that, before colliding, particles are uncorrelated. While this bold assumption can be motivated physically, it is not completely clear how it should be generalized when considering higher-order distribution functions. Our point is that if one considers the Stosszahlansatz as a heuristically motivated assumption, it generalizes naturally to higher-order cases-even though this raises extra mathematical challenges!

We start with a brief reminder on MaxEnt distributions as well as on the BBGKY hierarchy leading to kinetic equations, and bridge both in the last two sections.

\section{Maximum Entropy Distributions}

The Shannon entropy $H(X)=-\int d x P(x) \ln P(x)$ has all properties one would expect from an uncertainty measure $[13,14]$. In other terms, among a set of distributions, the least biased guess an observer can make is the one having the largest entropy while still satisfying available observational constraints. Assume for instance we try to maximize $H(X)$ under the constraint $\langle O\rangle=$ $\int d x O(x) P(x)=\mu$. Introducing a multiplier for the constraint and another for the probabilistic normalization, one has to compute

$$
\frac{\partial}{\partial P}\left(\int d x P(x) \ln \frac{1}{P(x)}+\lambda_{0} \int d x P(x)+\lambda \int d x O(x) P(x)\right)=\int d x\left(\ln \frac{1}{P(x)}-1+\lambda_{0}+\lambda O(x)\right) .
$$

Letting this expression vanish yields

$$
P(x)=\exp \left(-1+\lambda_{0}+\lambda O(x)\right)
$$

where the multipliers have to be determined to match the constraints. This result extends straightforwardly to the case of several constraints, namely $\left\langle O_{k}\right\rangle=\int d x O_{k}(x) P(x)=\mu_{k}$ for $k=1,2, \ldots, K$. Then

$$
P(x)=\frac{1}{Z} \exp \left(\sum_{k=1}^{K} \lambda_{k} O_{k}(x)\right) .
$$

In what follows we shall be concerned primarily with constraints on marginals instead of averages. An appropriate use of $\delta$ functions allows to generalize the previous result. As an example consider the case of a quadrivariate variable $\mathbf{x}=(w, x, y, z)$ the marginal $P_{123}(a, b, c)$ of which is assumed to be known. Putting $O(\mathbf{x})=\delta(w, a) \delta(x, b) \delta(y, c)$ we can write 


$$
\begin{aligned}
\langle O\rangle & =\sum_{\mathbf{x}} O(\mathbf{x}) P(\mathbf{x}) \\
& =\sum_{w, x, y} \delta(w, a) \delta(x, b) \delta(y, c) \sum_{z} P(\mathbf{x}) \\
& =\sum_{w, x, y} \delta(w, a) \delta(x, b) \delta(y, c) P_{123}(w, x, y) \\
& =P_{123}(a, b, c) .
\end{aligned}
$$

Using the result (3) derived for the averages one gets

$$
P(\mathbf{x})=\frac{1}{Z} \exp \left(\sum_{a, b, c} \lambda(a, b, c) O(\mathbf{x})\right)=\frac{1}{Z} \exp \lambda(w, x, y)
$$

$\lambda$ now denoting a function. This result can be extended to any number of marginals of any order. If for instance besides $P_{123}$ the marginals $P_{124}$ and $P_{34}$ are given we get

$$
P(\mathbf{x})=\frac{1}{Z} \exp \left(\lambda_{1}(w, x, y)+\lambda_{2}(w, x, z)+\lambda_{3}(y, z)\right)
$$

for functions $\lambda_{1}, \lambda_{2}$ and $\lambda_{3}$ to be determined. Unfortunately, this determination is difficult except in the case of univariate constraints. Then $\lambda_{1}(w)=\ln P_{1}(w)$, etc., obviously solves the problem, so that the corresponding MaxEnt distribution is the factorized distribution. When turning our attention to the applicability of this result to the realm of kinetic theory, it will appear that in that context the problem can be slightly simplified due to the structure of reduced distributions.

\section{The BBGKY Hierarchy}

Let us consider $N$ particles of mass $m$, the coordinates of which in phase space are their positions $\mathbf{q}_{i}$ and momenta $\mathbf{p}_{i}$. It will be convenient to define a condensed notation $x_{i}=\left(\mathbf{q}_{i}, \mathbf{p}_{i}\right)$. Let $f_{N}\left(x_{1}, \ldots, x_{N}, t\right)$ denote the joint distribution function characterizing the system, which obeys Liouville's equation

$$
\frac{d f_{N}}{d t}=\frac{\partial f_{N}}{\partial t}+\sum_{i=1}^{N} \frac{\mathbf{p}_{i}}{m} \frac{\partial f_{N}}{\partial \mathbf{q}_{i}}+\sum_{i=1}^{N} \mathbf{F}_{i} \frac{\partial f_{N}}{\partial \mathbf{p}_{i}}=0
$$

where $\mathbf{F}_{i}$ denotes the force exerted on particle $i$. We shall restrict ourselves to the case without external force and where particles interact pairwise through some radial potential $V\left(\left|\mathbf{q}_{i}-\mathbf{q}_{j}\right|\right)=V_{i j}$. Then $\mathbf{F}_{i}=-\sum_{j \neq i} \frac{\partial V_{i j}}{\partial \mathbf{q}_{i}}$.

Reminding that $f_{N}$ itself is normalized to $N$ !, we now introduce the reduced $s$-particle distribution $f_{s}\left(x_{1}, \ldots, x_{s}, t\right)=\frac{N !}{(N-s) !} \int d x_{s+1} \ldots d x_{N} f_{N}\left(x_{1}, \ldots, x_{N}, t\right)$. The standard result [15] is that by integrating Liouville's equation, one obtains a dynamical equation for $f_{s}$ given by the so-called BBGKY hierarchy (from the non-chronological list of its co-discoverers' names : Bogoliubov, Born, Green, Kirkwood, Yvon) :

$$
\frac{\partial f_{s}}{\partial t}+\sum_{i=1}^{s} \frac{\mathbf{p}_{i}}{m} \frac{\partial f_{s}}{\partial \mathbf{q}_{i}}-\sum_{i=1}^{s} \sum_{j \neq i}^{s} \frac{\partial V_{i j}}{\partial \mathbf{q}_{i}} \frac{\partial f_{s}}{\partial \mathbf{p}_{i}}-\int d x_{s+1} \sum_{i=1}^{s} \frac{\partial V_{i, s+1}}{\partial \mathbf{q}_{i}} \frac{\partial f_{s+1}}{\partial \mathbf{p}_{i}}=0
$$


This expression forms a hierarchy since the dynamics for $f_{s}$ is expressed in terms of the higher-order distribution $f_{s+1}$. Of course each equation can be deduced from its higher-order precursor by integration, at the cost of an information loss. In what follows we shall denote the $s$-th equation of the hierarchy as BBGKY-s. BBGKY1 and BBGKY2, in which we are primarily interested here, are

$$
\frac{\partial f_{1}}{\partial t}+\frac{\mathbf{p}_{1}}{m} \frac{\partial f_{1}}{\partial \mathbf{q}_{1}}=\int d x_{2} \frac{\partial V_{12}}{\partial \mathbf{q}_{1}} \frac{\partial f_{2}}{\partial \mathbf{p}_{1}}
$$

and

$$
\frac{\partial f_{2}}{\partial t}+\frac{\mathbf{p}_{1}}{m} \frac{\partial f_{2}}{\partial \mathbf{q}_{1}}+\frac{\mathbf{p}_{2}}{m} \frac{\partial f_{2}}{\partial \mathbf{q}_{2}}-\frac{\partial V_{12}}{\partial \mathbf{q}_{1}}\left(\frac{\partial}{\partial \mathbf{p}_{1}}-\frac{\partial}{\partial \mathbf{p}_{2}}\right) f_{2}=\int d x_{3}\left(\frac{\partial V_{13}}{\partial \mathbf{q}_{1}} \frac{\partial f_{3}}{\partial \mathbf{p}_{1}}+\frac{\partial V_{23}}{\partial \mathbf{q}_{2}} \frac{\partial f_{3}}{\partial \mathbf{p}_{2}}\right),
$$

where of course $f_{1}=f_{1}\left(\mathbf{p}_{1}, \mathbf{q}_{1}, t\right), f_{2}=f_{2}\left(\mathbf{p}_{1}, \mathbf{q}_{1}, \mathbf{p}_{2}, \mathbf{q}_{2}, t\right)$ and $f_{3}=f_{3}\left(\mathbf{p}_{1}, \mathbf{q}_{1}, \mathbf{p}_{2}, \mathbf{q}_{2}, \mathbf{p}_{3}, \mathbf{q}_{3}, t\right)$. The purpose of this paper is to investigate the second of these equations. As stressed above, we shall not try to express BBGKY1 and BBGKY2 as a set of coupled equations relating $f_{1}$ and $f_{2}$, since such an approach would not "fit" nicely in the spirit of the BBGKY approach. Instead, we shall manage to truncate BBGKY2 in order to obtain a single, self-standing equation for $f_{2}$.

\section{The Stosszahlansatz for BBGKY2}

The procedure leading from the BBGKY1 equation to a consistent kinetic equation for $f_{1}$ is standard: the Stosszahlansatz asserts that before colliding two particles are uncorrelated, i.e., $f_{2}$ factorizes as $f_{2}\left(x_{1}, x_{2}\right)=f_{1}\left(x_{1}\right) f_{1}\left(x_{2}\right)$. This allows us to express the collision integral in terms of $f_{1}$, so that BBGKY1 becomes a closed equation for $f_{1}$. Since this factorization hypothesis may be supported from a physical standpoint, it is tempting to use this ansatz in the collision term for BBGKY2 as well. But this raises an issue: if BBGKY2 can be cast into an equation relating a streaming term expressed in terms of $f_{2}$ to a collision term expressed in terms of $f_{1}$, then this equation is obviously not consistent by itself and has to be supplemented, so as to obtain a system of coupled equations.

Our point is that this issue vanishes if the Stosszahlansatz is reconsidered as a heuristic ansatz instead of a physically-grounded assumption. We propose to reformulate it as follows: since the exact codependence of particles entering the collision range is unknown, one has to make a reasonable guess on it, and the MaxEnt distribution steps out at this point. The MaxEnt guess for $f_{2}$, compatible with the univariate distribution appearing in the streaming term, is the factorized one, but on the contrary the guess for $f_{3}$, compatible with the $f_{2}$ appearing in the left-hand side, will be quite different from a factorized distribution (as exemplified by (6)).

Let us now see to what extent the result (6) obtained for the MaxEnt distribution may be particularized to our current purpose. We showed that, given bivariate marginals, the MaxEnt estimate for $f_{3}\left(x_{1}, x_{2}, x_{3}\right)$ was given by

$$
f_{3}^{M E}\left(x_{1}, x_{2}, x_{3}\right)=\frac{1}{Z} \exp \left(\lambda_{1}\left(x_{1}, x_{2}\right)+\lambda_{2}\left(x_{1}, x_{3}\right)+\lambda_{3}\left(x_{2}, x_{3}\right)\right)
$$

for some $\lambda_{1}, \lambda_{2}$ and $\lambda_{3}$. The point is that these marginals are the same for each pair by definition of the reduced distribution $f_{2}$, and accordingly all three $\lambda$ s are the same. Absorbing the normalization, one is therefore allowed to write that

$$
f_{3}^{M E}\left(x_{1}, x_{2}, x_{3}\right)=g\left(x_{1}, x_{2}\right) g\left(x_{1}, x_{3}\right) g\left(x_{2}, x_{3}\right)
$$


for a function $g$ that is nevertheless unknown, except for the fact that it has to satisfy the marginal constraint

$$
g(x, y) \int d z g(x, z) g(y, z)=f_{2}(x, y)
$$

\section{The Collision Term}

Using this ansatz we now proceed to write down the kinetic equation for $f_{2}$. All through, we shall retain the usual assumptions of kinetic theory, leading us to neglect triple collisions: the streaming term for the two-particle distribution characterizing particles " 1 " and " 2 " will thus be altered by (1) binary collisions between " 1 " and another particle, "2" being spectator, and (2) binary collisions between "2"and another particle, "1" being spectator.

The binary interaction is defined as occurring when two particles meet in a ball $B$ of radius $R$. Defining ternary interactions is more subtle since, inasmuch as the interaction potential is the same whatever the order of the interaction, it seems artificial to introduce a specific cutoff. We shall therefore define the range of triple collisions as the lenticular overlap of balls $B_{R}^{(1)}$ and $B_{R}^{(2)}$ characterizing the domain of interaction with " 1 " and " 2 " respectively. Neglecting triple collisions thus amounts to assuming that $\left|\mathbf{q}_{1}-\mathbf{q}_{2}\right|>2 R$.

We first compute the contribution of collisions of " 2 " with " 3 ", " 1 " being left aside. Let us recall that the collision term is given by

$$
\left(\frac{\partial f_{2}}{\partial t}\right)_{\text {coll }}=\int d x_{3}\left(\frac{\partial V_{13}}{\partial \mathbf{q}_{1}} \frac{\partial f_{3}}{\partial \mathbf{p}_{1}}+\frac{\partial V_{23}}{\partial \mathbf{q}_{2}} \frac{\partial f_{3}}{\partial \mathbf{p}_{2}}\right)
$$

In the usual derivation of the Boltzmann equation from the BBGKY hierarchy, the right-hand side of BBGKY1 is transformed using BBGKY2. Similarly, we can transform $\left(\partial_{t} f_{2}\right)_{\text {coll }}$ using BBGKY3, namely

$$
\begin{aligned}
& \frac{\partial f_{3}}{\partial t}+\frac{\mathbf{p}_{1}}{m} \frac{\partial f_{3}}{\partial \mathbf{q}_{1}}+\frac{\mathbf{p}_{2}}{m} \frac{\partial f_{3}}{\partial \mathbf{q}_{2}}+\frac{\mathbf{p}_{3}}{m} \frac{\partial f_{3}}{\partial \mathbf{q}_{3}} \\
& \quad-\frac{\partial V_{12}}{\partial \mathbf{q}_{1}}\left(\frac{\partial}{\partial \mathbf{p}_{1}}-\frac{\partial}{\partial \mathbf{p}_{2}}\right) f_{3}-\frac{\partial V_{13}}{\partial \mathbf{q}_{1}}\left(\frac{\partial}{\partial \mathbf{p}_{1}}-\frac{\partial}{\partial \mathbf{p}_{3}}\right) f_{3}-\frac{\partial V_{23}}{\partial \mathbf{q}_{2}}\left(\frac{\partial}{\partial \mathbf{p}_{2}}-\frac{\partial}{\partial \mathbf{p}_{3}}\right) f_{3}=\left(\frac{\partial f_{3}}{\partial t}\right)_{c o l l}
\end{aligned}
$$

(we do not make explicit the collision term $\left(\partial_{t} f_{3}\right)_{\text {coll }}$ since we shall cancel it soon anyway). Under usual dimensional assumptions, we can write $\partial_{t} f_{3} \approx 0$ and $\left(\partial_{t} f_{3}\right)_{\text {coll }} \approx 0$, so that, substituting in the collision term, $\left(\partial_{t} f_{2}\right)_{\text {coll }}$ is rewritten as

$$
\begin{aligned}
\left(\frac{\partial f_{2}}{\partial t}\right)_{\text {coll }} & =\int d x_{3}\left(\frac{\mathbf{p}_{1}}{m} \frac{\partial f_{3}}{\partial \mathbf{q}_{1}}+\frac{\mathbf{p}_{2}}{m} \frac{\partial f_{3}}{\partial \mathbf{q}_{2}}+\frac{\mathbf{p}_{3}}{m} \frac{\partial f_{3}}{\partial \mathbf{q}_{3}}-\frac{\partial V_{12}}{\partial \mathbf{q}_{1}}\left(\frac{\partial}{\partial \mathbf{p}_{1}}-\frac{\partial}{\partial \mathbf{p}_{2}}\right) f_{3}+\left(\frac{\partial V_{13}}{\partial \mathbf{q}_{1}}+\frac{\partial V_{23}}{\partial \mathbf{q}_{2}}\right) \frac{\partial f_{3}}{\partial \mathbf{p}_{3}}\right) \\
& =\int d x_{3}\left(\frac{\mathbf{p}_{2}}{m} \frac{\partial f_{3}}{\partial \mathbf{q}_{2}}+\frac{\mathbf{p}_{3}}{m} \frac{\partial f_{3}}{\partial \mathbf{q}_{3}}\right)
\end{aligned}
$$

(the last term vanishes due to the boundary condition $f_{3}(|\mathbf{p}| \rightarrow \infty)=0$, the penultimate since "1" and " 2 " are supposed far apart from each other and the first because $f_{3}$ depends but weakly on $\mathbf{q}_{1}$ ). More precisely,

$$
\left(\frac{\partial f_{2}}{\partial t}\right)_{\text {coll }}=\int_{\mathbf{q}_{3} \in B_{R}^{(2)}} d \mathbf{q}_{3} d \mathbf{p}_{3}\left(\frac{\mathbf{p}_{2}}{m} \frac{\partial f_{3}}{\partial \mathbf{q}_{2}}+\frac{\mathbf{p}_{3}}{m} \frac{\partial f_{3}}{\partial \mathbf{q}_{3}}\right) .
$$


The following is standard [15]. We introduce the relative coordinate $\mathbf{r}_{23}=\mathbf{q}_{3}-\mathbf{q}_{2}$ and use Gauss' theorem in order to rewrite $\left(\partial_{t} f_{2}\right)_{\text {coll }}$ as a surface integral, so that

$$
\begin{aligned}
\left(\frac{\partial f_{2}}{\partial t}\right)_{\text {coll }} & =\int_{\mathbf{r}_{23} \in B_{R}} d \mathbf{r}_{23} d \mathbf{p}_{3} \frac{\mathbf{p}_{3}-\mathbf{p}_{2}}{m} \frac{\partial}{\partial \mathbf{r}_{23}} f_{3}\left(\mathbf{q}_{1}, \mathbf{p}_{1}, \mathbf{q}_{2}, \mathbf{p}_{2}, \mathbf{q}_{3}, \mathbf{p}_{3}, t\right) \\
& =\int_{S_{R}} d \mathbf{p}_{3} d \Sigma \cdot \frac{\mathbf{p}_{3}-\mathbf{p}_{2}}{m} f_{3}\left(\mathbf{q}_{1}, \mathbf{p}_{1}, \mathbf{q}_{2}, \mathbf{p}_{2}, \mathbf{q}_{3}, \mathbf{p}_{3}, t\right) \\
& =\int_{S_{R}^{-} \cup S_{R}^{+}} d \mathbf{p}_{3} d \Sigma \cdot \frac{\mathbf{p}_{3}-\mathbf{p}_{2}}{m} f_{3}\left(\mathbf{q}_{1}, \mathbf{p}_{1}, \mathbf{q}_{2}, \mathbf{p}_{2}, \mathbf{q}_{3}, \mathbf{p}_{3}, t\right),
\end{aligned}
$$

where $d \Sigma$ denotes the surface element of the sphere $S_{R}$ such that $\left|\mathbf{r}_{23}\right|=R$. The southern hemisphere is interpreted as the contribution of oncoming collisions since $\left(\mathbf{p}_{3}-\mathbf{p}_{2}\right) \cdot d \Sigma<0$, while the northern one is the contribution of ending collisions since $\left(\mathbf{p}_{3}-\mathbf{p}_{2}\right) \cdot d \Sigma>0$.

Orienting the polar axis along $\mathbf{p}_{3}-\mathbf{p}_{2}$, we have $d \Sigma \cdot\left(\mathbf{p}_{3}-\mathbf{p}_{2}\right)=\left|\mathbf{p}_{3}-\mathbf{p}_{2}\right| R^{2} \sin \theta \cos \theta d \theta d \phi$. This can be re-expressed in terms of the surface element of the azimuthal plane such that $\theta=\pi / 2$. Letting $r$ denote the radial component on the plane, we obviously have $r=R \sin \theta$, whence $d r= \pm R \cos \theta d \theta$ (depending on $\theta$ being lesser or larger than $\pi / 2)$ and $d \Sigma \cdot\left(\mathbf{p}_{3}-\mathbf{p}_{2}\right)= \pm\left|\mathbf{p}_{3}-\mathbf{p}_{2}\right| d \omega$. The collision term can thus be rewritten as (approximating $\mathbf{q}_{3} \approx \mathbf{q}_{2}$ )

$$
\begin{aligned}
\left(\frac{\partial f_{2}}{\partial t}\right)_{\text {coll }}= & \int_{\text {after }} d \mathbf{p}_{3} d \omega \frac{\left|\mathbf{p}_{3}-\mathbf{p}_{2}\right|}{m} f_{3}\left(\mathbf{q}_{1}, \mathbf{p}_{1}, \mathbf{q}_{2}, \mathbf{p}_{2}, \mathbf{q}_{2}, \mathbf{p}_{3}, t\right) \\
& -\int_{\text {before }} d \mathbf{p}_{3} d \omega \frac{\left|\mathbf{p}_{3}-\mathbf{p}_{2}\right|}{m} f_{3}\left(\mathbf{q}_{1}, \mathbf{p}_{1}, \mathbf{q}_{2}, \mathbf{p}_{2}, \mathbf{q}_{2}, \mathbf{p}_{3}, t\right)
\end{aligned}
$$

Now comes the ansatz. Before the collision we obviously have

$$
f_{3}\left(\mathbf{q}_{1}, \mathbf{p}_{1}, \mathbf{q}_{2}, \mathbf{p}_{2}, \mathbf{q}_{2}, \mathbf{p}_{3}, t\right)=g\left(\mathbf{q}_{1}, \mathbf{p}_{1}, \mathbf{q}_{2}, \mathbf{p}_{2}, t\right) g\left(\mathbf{q}_{1}, \mathbf{p}_{1}, \mathbf{q}_{2}, \mathbf{p}_{3}, t\right) g\left(\mathbf{q}_{2}, \mathbf{p}_{2}, \mathbf{q}_{2}, \mathbf{p}_{3}, t\right)
$$

The ansatz may be extended after the collision using the fact that, by Liouville's equation, $f_{3}\left(\mathbf{q}_{1}, \mathbf{p}_{1}, \mathbf{q}_{2}, \mathbf{p}_{2}, \mathbf{q}_{2}, \mathbf{p}_{3}, t\right)=f_{3}\left(\mathbf{q}_{1}^{-\tau}, \mathbf{p}_{1}, \mathbf{q}_{2}^{-\tau}, \mathbf{p}_{2}^{\prime}, \mathbf{q}_{2}^{-\tau}, \mathbf{p}_{3}^{\prime}, t-\tau\right)$, where $\tau$ is the retardation such that at $t-\tau$ the particles are entering the collision range with momenta $\mathbf{p}_{2}^{\prime}, \mathbf{p}_{3}^{\prime}$. Since $\mathbf{q}_{i}^{-\tau} \approx \mathbf{q}_{i}$ and $t \approx t-\tau$, and since $\mathbf{p}_{2}^{\prime}, \mathbf{p}_{3}^{\prime}$ are pre-collisional momenta, the ansatz may be introduced in the first integral as well with arguments $\mathbf{p}_{2}^{\prime}, \mathbf{p}_{3}^{\prime}$. We are therefore eventually led to the following Boltzmann-like form for the BBGKY2 equation:

$$
\begin{aligned}
\frac{\partial f_{2}}{\partial t}+ & \frac{\mathbf{p}_{1}}{m} \frac{\partial f_{2}}{\partial \mathbf{q}_{1}}+\frac{\mathbf{p}_{2}}{m} \frac{\partial f_{2}}{\partial \mathbf{q}_{2}}-\frac{\partial V_{12}}{\partial \mathbf{q}_{1}}\left(\frac{\partial}{\partial \mathbf{p}_{1}}-\frac{\partial}{\partial \mathbf{p}_{2}}\right) f_{2} \\
= & \int d \mathbf{p}_{3} d \omega \frac{\left|\mathbf{p}_{3}-\mathbf{p}_{2}\right|}{m}\left(g\left(\mathbf{q}_{1}, \mathbf{p}_{1}, \mathbf{q}_{2}, \mathbf{p}_{2}^{\prime}\right) g\left(\mathbf{q}_{1}, \mathbf{p}_{1}, \mathbf{q}_{2}, \mathbf{p}_{3}^{\prime}\right) g\left(\mathbf{q}_{2}, \mathbf{p}_{2}^{\prime}, \mathbf{q}_{2}, \mathbf{p}_{3}^{\prime}\right)\right. \\
& \left.-g\left(\mathbf{q}_{1}, \mathbf{p}_{1}, \mathbf{q}_{2}, \mathbf{p}_{2}\right) g\left(\mathbf{q}_{1}, \mathbf{p}_{1}, \mathbf{q}_{2}, \mathbf{p}_{3}\right) g\left(\mathbf{q}_{2}, \mathbf{p}_{2}, \mathbf{q}_{2}, \mathbf{p}_{3}\right)\right) \\
& +(1 \leftrightarrow 2)
\end{aligned}
$$

The last term accounts for the contribution of collisions undergone by particle "1". 


\section{Final Remarks}

As promised, equation (21) is coherent for $f_{2}$ since $g$ can-in principle-be solved in terms of $f_{2}$. Unfortunately, we are not aware of any readily available solution to the integral equation (13). It is interesting to note that kinetic theory provides a motivation for studying the mathematical object (12), which proves surprisingly involved in spite of its deceptive apparent simplicity. In the authors' opinion, it might turn sound to tackle the problem from a linearized vantage point, considering situations where the particles are almost uncorrelated, that is where $g$ is almost factorizable. Such an approach would also be in line with usual methods of kinetic theory [16].

This current implicit form of the collision term bears a close ressemblance with the one appearing in the standard Boltzmann equation. This ressemblance might however turn deceptive since $g$ is likely to be a complicated functional of $f_{2}$. We hope that the present letter can prompt further work on this collision term, which might eventually lead to a form susceptible of a hydrodynamical treatment. Our hope is that such a treatment could lead to a deeper understanding of fluctuating phenomena, for which the first-order theory provides only lacunary insights. In particular, it seems reasonable to expect that (21) can be cast in the lattice-based formalism which proved so successful for the usual Boltzmann equation.

\section{Acknowledgments}

The authors would like to acknowledge funding from the European Union Seventh Framework Programme, under grant agreement 317534 (Sophocles).

\section{Author Contributions}

G.C., O.M. and B.C. performed the research; B.C. supervised the project; G.C. wrote the manuscript. All authors have read and approved the final manuscript.

\section{Conflicts of Interest}

The authors declare no conflict of interest.

\section{References}

1. Jaynes, E.T. Information Theory and Statistical Mechanics. Phys. Rev. 1957, 106, doi:10.1103/ PhysRev.106.620.

2. Jaynes, E.T. Information Theory and Statistical Mechanics. II. Phys. Rev. 1957, 108, doi:10.1103/PhysRev.108.171.

3. Schneidman, E.; Still, S.; Berry, M.J.; Bialek, W. Network Information and Connected Correlations. Phys. Rev. Lett. 2003, 91, 238701.

4. Schneidman, E.; Berry, M.J.; Segev, R.; Bialek, W. Weak Pairwise Correlations Imply Strongly Correlated Network States in a Neural Population. Nature 2006, 440, 1007-1012.

5. Stephens, G.J.; Bialek, W. Statistical Mechanics of Letters in Words. Phys. Rev. E 2010, 81, 066119. 
6. Mora, T.; Bialek, W. Are Biological Systems Poised at Criticality ? J. Stat. Phys. 2011, 144, 268-302.

7. Bialek, W.; Cavagna, A.; Giardina, I.; Mora, T.; Silvestri, E.; Viale, M.; Walczak, A.M. Statistical Mechanics for Natural Flocks of Birds. Proc. Natl. Acad. Sci. USA 2012, 109, 4786-4791.

8. Stephens, G.J.; Mora, T.; Tkačik, G.; Bialek, W. Statistical Thermodynamics of Natural Images. Phys. Rev. Lett. 2013, 110, 018701.

9. Van der Straeten, E. Maximum Entropy Estimation of Transition Probabilities of Reversible Markov Chains. Entropy 2009, 11, 867-887.

10. Marre, O.; El Boustani, S.; Frégnac, Y.; Destexhe, A. Prediction of Spatiotemporal Patterns of Neural Activity from Pairwise Correlations. Phys. Rev. Lett. 2009, 102, 138101.

11. Cavagna, A.; Giardina, I.; Ginelli, F.; Mora, T.; Piovani, D.; Tavarone, R.; Walczak, A.M. Dynamical Maximum Entropy Approach to Flocking. Phys. Rev. E 2014, 89, 042707.

12. Chliamovitch, G.; Dupuis, A.; Golub, A.; Chopard, B. Improving Predictability of Time Series Using Maximum Entropy Methods. Europhys. Lett. 2015, 110, doi:10.1209/0295-5075/110/ 10003.

13. Shannon, C.E. A Mathematical Theory of Communication. Bell Syst. Tech. J. 1948, 27, 379-423.

14. Khinchin, A.Y. Mathematical Foundations of Information Theory; Dover: Mineola, NY, USA, 1957.

15. Kreuzer, H.J. Nonequilibrium Thermodynamics and its Statistical Foundations; Oxford University Press: Oxford, UK, 1984.

16. Liboff, R.L. Kinetic Theory; Springer: New York, NY, USA, 2003.

(c) 2015 by the authors; licensee MDPI, Basel, Switzerland. This article is an open access article distributed under the terms and conditions of the Creative Commons Attribution license (http://creativecommons.org/licenses/by/4.0/). 